
\title{
28 Research Soure \\ EV-A71 Induced IL-1Beta Production in THP-1 Macrophages is Dependent on NLRP3, RIG-I, and TLR3
}

Hsing-l Huang ( $\boldsymbol{\nabla}$ hihuang@mail.cgu.edu.tw)

Chang Gung University

Chi-Chong Chio

Chang Gung University

Jhao-Yin Lin

Chang Gung University

Chia-Jung Chou

Chang Gung University

Chia-Chen Lin

Chang Gung University

Liang-Sheng Yu

Chang Gung University

\section{Research Article}

Keywords:

Posted Date: January 25th, 2022

DOI: https://doi.org/10.21203/rs.3.rs-1246588/v1

License: @ (1) This work is licensed under a Creative Commons Attribution 4.0 International License. Read Full License 


\section{Abstract}

Enterovirus A71 (EV-A71) is an emerging enterovirus that can cause neurological complications in young children. The severity of EV71 infection is correlated with the production of cytokines, including IL-6 and IL-1b. Macrophages are specialized immune cells that have been deemed the major cell source for IL-1b. The production of IL- $1 \mathrm{~b}$ is a result of inflammasome activation, an innate immune response that involves the assembly of multiple proteins. However, the mechanisms by which EV-A71 activates inflammasomes are not fully understood. Here, we confirmed that THP-1 macrophages permissive EV-A71 replication. EVA71 infection is sufficient to trigger IL-1b production in macrophages. Knockdown of NLRP3, RIG-I and TLR3 in macrophages suppressed IL-1b secretion. Moreover, caspase-1 and caspase-8 activation are involved in IL-1b production. In summary, this study showed that NLRP3 and the RNA sensors TLR3 and RIG-I are implicated in EV-A71-induced inflammasome activation in macrophages to facilitate IL-1b production via activated caspase-1 and caspase-8.

\section{Introduction}

EV-A71 is a positive-sense RNA virus that belongs to the member of the family Picornaviridae. EV-A71 infection is associated with several diseases, including hand, foot, and mouth disease (HFMD) and herpangina. Moreover, EV-A71 may invade the central nervous system and cause severe neurological complications, causing significant concern in the Asia-Pacific region ${ }^{1}$. The severity of EV-A71 infection has been suggested to be correlated with the production of pro-inflammatory cytokines ${ }^{2}$. Previous studies suggested that the production of inflammatory cytokines such as IL- 6 and IL-1 $\beta$ may be involved in central nervous system (CNS) damage caused by EV-A71 infection ${ }^{3,4}$.

As the major effector cells of the innate immune system, macrophages play essential roles in the recognition and destruction of invading microorganisms. When exposed to pathogens or inflammatory stimuli, they release cytokines and chemokines to induce enhanced vascular permeability and recruitment of immune cells ${ }^{5}$. However, many viruses are able to infect and replicate in macrophages to facilitate their dissemination ${ }^{6}$. Macrophage infection induces the expression of cytokines, which is associated with disease severity ${ }^{7}$. Moreover, emerging evidence suggests that virus infection can activate inflammasomes in macrophages to produce IL-1 $\beta$ and IL-18 $8^{8,9}$. Excessive IL-1 $\beta$ expression is associated with Theiler's murine encephalomyelitis virus (TMEV)-induced demyelinating disease by promoting the generation of Th17 cells ${ }^{10}$. Furthermore, a recent study indicated that the nucleotide-binding oligomerization domain 3 (NLRP3) inflammasome is involved in neuroinvasion and neuroinflammation during severe acute respiratory syndrome coronavirus-2 (SARS-CoV-2) infection ${ }^{11}$. Inflammasomes are composed of multiple proteins, including NOD-like receptors (NLRs), the adaptor protein apoptosisassociated speck-like protein containing caspase recruitment domain (CARD) known as ASC, and the effector protein pro-caspase-1. Recently, several types of inflammasomes were characterized, and it was revealed that NLRP1, NLRP3, NLR family CARD domain-containing 4 (NLRC4) and pyrin inflammasome 
activation is mediated by NLR family members, while the absent in melanoma 2 (AIM2) inflammasome is activated by AIM2, which belongs to the IfI202/IFI116 family ${ }^{12,13}$.

RNA viruses such as IAV, human immunodeficiency virus (HIV), and encephalomyocarditis virus (EMCV) can induce NLRP3 inflammasome activation ${ }^{14-16}$ and the subsequent generation of IL-1 $\beta$. The formation of NLRP3 inflammasomes has been demonstrated to be involved in viral pathogenesis ${ }^{17-19}$. The NLRP3 inflammasome can be activated by a wide range of stimuli, such as extracellular ATP, asbestos, silica, alum, amyloid $\beta$, single-stranded RNA (ssRNA), double-stranded (dsRNA) analogs, and pathogen products $^{20}$. Accumulating evidence has demonstrated that picornaviruses, including EMCV, coxsackievirus B3 (CVB3), and poliovirus (PV), can produce IL-1 $\beta^{16,21}$. The induction of IL-1 $\beta$ is implicated in CVB3-induced myocarditis, and the transplantation of NLRP3-knockdown (KD) macrophages results in reduced IL- $\beta$ secretion and milder symptoms ${ }^{21}$, indicating that IL-1 $\beta$ plays an essential role in pathogenesis. In human rhinovirus (HRV)-infected airway epithelial cells, inflammasomedependent IL-1 $\beta$ secretion is involved in the exacerbation of pulmonary symptoms ${ }^{22}$. Interestingly, the production of IL-1 $\beta$ can result in the pyroptosis of EV-A71-infected neural cells, which suggests that inflammasome activation may be involved in programmed cell death ${ }^{3}$. In contrast, the production of IL-1 $\beta$ has been reported to play beneficial roles during enteroviral infections. For example, Wang et al. showed that CVB3 infection resulted in more severe symptoms in NLRP3-knockout mice ${ }^{23}$, while the NLRP3 inflammasome was demonstrated to elicit beneficial effects on EV71-infected animals ${ }^{24}$. The conflicting results of these studies suggest that inflammasome activation may exacerbate or ameliorate disease severity during enteroviral infections.

Previous research has shown that EV-A71 infection can activate IL-1 $\beta$ release mediated by the NLRP3 inflammasome activated via viral proteins ${ }^{24,25}$. However, the mechanisms associated with EV-A71induced IL-1 $\beta$ production in macrophages have not been completely characterized. In this study, we demonstrated that in addition to NLRP3, PRRs, including RIG-I and TLR-3, are involved in inflammasome activation in EV-A71-infected THP-1 macrophages. Moreover, both caspase-1 and caspase-8 are implicated in EV-A71-induced IL-1 $\beta$ production in THP-1 macrophages. Our results suggest that the release of IL-1 $\beta$ is modulated by multiple PRRs and caspases in THP-1 macrophages.

\section{Methods}

\section{Cells and virus}

THP-1 cells were cultured in RPMI-1640 medium (Thermo-Fisher Scientific, MA, USA) containing 10\% fetal bovine serum (FBS) (GE, Boston, USA). Human rhabdomyosarcoma (RD) cells were obtained from Dr. Shih Shin-Ru and grown in Dulbecco modified Eagle medium (DMEM) supplemented with 10\% FBS, $1 \%$ non-essential amino acids, 1\% L-glutamine, and 1\% penicillin/streptomycin (all from Thermo-Fisher Scientific, MA, USA). NLRP3-KD THP-1 cells (were gifted by Dr. Jan Martel, Chang Gung University, Immunology Center) are THP-1 cells that infected with lentiviral vectors that carried NLRP3 shRNA. All 
cells were incubated in a $37^{\circ} \mathrm{C}$ incubator that equilibrated with $5 \% \mathrm{CO}_{2}$. The EV-A71 strain 2231

(TW/2231/98), strain 70811 (TW/70811/08) was isolated from the Clinical Virology Laboratory of Chang Gung Memorial Hospital (Linkou, Taiwan), as well as EV-A71 strain $\mathrm{BrCr}$ (USA/BrCr/1970), was amplified using RD cells, and the titer was quantified by plaque forming assays.

\section{Isolation of PBMCs and differentiation of PBMCs toward macrophages}

Human peripheral blood samples were collected after approval by IRB (Chang Gung Medical Foundation institutional review board, IRB2019050059). All the research process was performed in accordance with IRB guidelines and regulations. The informed consent was obtained from all subjects and/or their legal guardians. Mononuclear cells were harvested by Ficoll-Paque method. Briefly, peripheral blood was mixed with PBS (1:1). The diluted cell suspension was then layered on Ficoll-Paque (GE Healthcare Life Sciences, MA, USA) (volume 2:1) in a $50 \mathrm{~mL}$ canonical tube. After centrifugation at $400 \mathrm{xg}$ for 20 minutes, the top layer was aspirated. The mononuclear cell layer in the interface was then transferred to a new tube. To differentiate the harvested PBMCs toward macrophages, RPMI medium supplemented with $20 \mathrm{ng} / \mathrm{mL}$ M-CSF (Peprotech, NJ, USA) and 1\% human serum was added and cultured for 5 days.

\section{Reagents}

$1 \mu \mathrm{g} / \mathrm{mL}$ LPS (Sigma-Alderich, MO, USA) was used to stimulate cells for activation. phorbol 12-myristate 13-acetate (PMA) was prepared at $200 \mu \mathrm{M}$ in DMSO (Dimethyl sulfoxide). $200 \mathrm{nM}$ PMA was used to stimulate THP-1 cells differentiation. Ac-YVAD-cmk (Sigma-Alderich, MO, USA) was prepared at $10 \mathrm{mM}$ in DMSO. $1 \mu \mathrm{M}$ Ac-YVAD-cmk was used to inhibit caspase-1 activation. Z-IETD-FMK (R\&D systems, MN, USA) was prepared at $20 \mathrm{mM}$ in DMSO. $20 \mu \mathrm{M}$ Z-IETD-FMK was used to treat PMA primed THP-1 after virus infection.

\section{Virus infection}

Cells were seeded in culture plates and cultivated at different time points. The seeded cells were rinsed by PBS twice and serum-free medium was added with the virus at a specified multiplicity of infection (M.O.I.). Adsorption was performed at $37^{\circ} \mathrm{C}$ for 1 hour, and the medium was then decanted. The cells were washed by PBS twice and 2\% FBS containing medium was added for further incubation.

\section{RNA extraction and RT-qPCR}

Trizol reagent (Life Technologies, Gaithersburg, MD) was applied to extract total RNA from cell samples. The cells were lysis by Trizol reagent and then mixed with chloroform. After $5 \mathrm{~min}$, the homogenate was centrifugated at $12,000 \mathrm{xg}$ for $15 \mathrm{~min}$ at $4^{\circ} \mathrm{C}$. The aqueous phase was transferred to a new tube and added with an equal volume of isopropanol and then incubated for $10 \mathrm{~min}$. The mixture was centrifuged at $12,000 \mathrm{xg}$ for $8 \mathrm{~min}$ at $4^{\circ} \mathrm{C}$. After the centrifugation, the supernatant was removed. $500 \mu \mathrm{L} 7 \%$ ethanol was used to wash the RNA pellet then centrifugated at $12,000 \times \mathrm{g}$ for $5 \mathrm{~min}$ at $4{ }^{\circ} \mathrm{C}$. Discard the supernatant and then air dried the RNA pellet. The RNA pellet was dissolved by sterile water and 
quantified the concertation of RNA by NonoDrop technology (Thermo-Fisher Scientific, MA, USA). $1 \mu \mathrm{g}$ of total RNA was used to synthesize cDNA. RevertAid First Strand cDNA Synthesis Kit (Thermo-Fisher Scientific, MA, USA) was ap-plied according to the manufacturer's instructions. To detect the expression of target genes, specific primers for TNF-a, IL-6, IL-1 $\beta$, EV-A71 5'UTR were used (Table 1). qPCR assays were carried out on 384-well plates and analyzed by a Roche Lightcycler 480 instrument (Roche, Basel, SW). Triplicate for each sample in qPCR analysis and 18s rRNA was used as a reference gene. The relative expression level of each gene was analyzed by $2^{-\triangle \Delta C T}$ method.

Table 1

qPCR primer used in this study.

\begin{tabular}{|c|c|c|}
\hline Gene & & sequence \\
\hline \multirow[t]{2}{*}{ Human 18s rRNA } & Forward & 5'- GTA ACC CGT TGA ACC CCA TT -3' \\
\hline & Reverse & 5'- CCA TCC AAT CGG TAG TAG CG -3' \\
\hline \multirow[t]{2}{*}{ EV-A71 5'UTR } & Forward & 5'- CCC TGA ATG CGG CTA ATC C -3' \\
\hline & Reverse & 5'- ATT GTC ACC ATA AGC AGC CA -3' \\
\hline \multirow[t]{2}{*}{ Human TNF-a } & Forward & 5'- CAT GAT CCG GGA CGT GGA GC -3' \\
\hline & Reverse & 5'- CTG ATT AGA GAG AGG TCC CTG -3' \\
\hline \multirow[t]{2}{*}{ Human IL-6 } & Forward & 5'- GGA GAC TTG CCT GGT GAA AA - $3^{\prime}$ \\
\hline & Reverse & 5'- GTC AGG GGT GGT TAT TGC AT -3' \\
\hline \multirow[t]{2}{*}{ Human IL-1 $\beta$} & Forward & 5'- ACA GAT GAA GTG CTC CTT CCA -3' \\
\hline & Reverse & 5'- GTC GGA GAT TCG TAG CTG GAT -3' \\
\hline
\end{tabular}

\section{Protein isolation and Western blot}

The cultured cells were harvested at indicated time point. After washed by PBS twice, the cell lysates were collected using protein lysis buffer ( $1 \%$ NP-40, $50 \mathrm{mM}$ Tris, and $150 \mathrm{mM} \mathrm{NaCl})$ containing protease inhibitors cocktail (Bioshop, Ontario, Canada). After incubated on ice for $30 \mathrm{~min}$, centrifugation was performed at $13,000 \mathrm{rpm}$ for $10 \mathrm{~min}$ at $4^{\circ} \mathrm{C}$. The Supernatants were harvested, and protein concentrations were measured by Protein Assay Dye Reagent (Bio-Rad Laboratories, CA, USA). Proteins $(30 \mu \mathrm{g})$ were separated by $1 \%$ or $1 \%$ SDS-polyacrylamide gel electrophoresis, and then transferred onto a poly-vinylidene fluoride membrane (PVDF) (GE, MA, USA). The membrane was then blocked with $5 \%$ skim milk in Tris-buffered saline Tween-20 buffer (TBST) $(20 \mathrm{mmol} / \mathrm{L}$ Tris- $\mathrm{HCl}, \mathrm{pH} 7.4,150 \mathrm{mmol} / \mathrm{L} \mathrm{NaCl}$, and $0 . \%$ Tween 20) at room temperature for one hour. After washed by TBST for twice, the membrane was then incubated with anti-EV-A71 3D Ab (1:10000, was gifted by Professor Shih Shin-Ru), anti-EV-A71 VP0 (1:1000, Millipore, MA, USA), anti-IL1 $\beta$ Ab (1:1000, Cell Signaling Technology, MA, USA), anti-NLRP3 Ab (1:1000, Cell Signaling Technology, MA, USA), anti-caspase-1 Ab (1:1000, Santa Cruz Biotechnology, TX, USA), anti-caspase-8 Ab (1:1000, Cell Signaling Technology, MA, USA), Anti-RIG-I Ab (1:1000, Enzo Life 
Sciences, NY, USA), anti-TLR3 Ab (1:1000, Abcam, CAMB, UK), anti-p-P65 Ab (1:1000, Cell Signaling Technology, MA, USA), anti-lamin Ab (1:1000, Santa Cruz Biotechnology, TX, USA). The unbound antibodies were then removed by washing with TBST three times. Horseradish peroxidase conjugated secondary antibodies (1:5000, Jackson ImmunoResearch Laboratories, Pennsylvania, USA) were incubated for 1 hour. Finally, the target proteins were visualized with a Western Lightning Chemiluminescence reagent (PerkinElmer, MA, USA) and detected by ChemiDoc imaging system (Bio-Rad Laboratories, CA, USA).

\section{siRNA transfection}

The siRNAs targeting for NLRP3, caspase-1, RIG-I, and TLR3, as well as scramble siRNA, were used in this study (all from Sigma-Aldrich, MO, USA). The siRNA was prepared in $100 \mu \mathrm{M}$ with RNase-free distilled water. $100 \mathrm{nM}$ siRNA was used to transfect into the cells with Lipofectamine RNAiMAX (Thermo-Fisher Scientific, MA, USA) in opti-MEM medium (Thermo-Fisher Scientific, MA, USA) for 48 hr. After transfection, the cells were infected with EV-A71 and harvested the sample at various hours post-infection.

\section{plaque assay}

The total virus was harvested at different time points after virus infection. RD cells were seeded on 6-well plate at a density of $5 \times 10^{5}$ cell per well. After incubation, the collected virus was serially diluted by $0 \%$ FBS DMEM medium. RD cells were washed by PBS twice then infected by diluted virus medium. After absorption, the medium was removed and replaced by $2 \%$ FBS DMEM medium containing $0.3 \%$ agarose. After 96 hours of incubation, the medium was removed and the cells were stained by crystal violet.

\section{ELISA}

The culture medium was collected at different time points. Centrifugation was performed at 5,000 rpm for 10 min at $4{ }^{\circ} \mathrm{C}$ then the supernatants were harvested. IL-1 $\beta$ ELISA kit was obtained from Invitrogen (Thermo-Fisher Scientific, MA, USA). The an-ti-IL-1 $\beta$ antibody was coating on the 96 well plate overnight at $4{ }^{\circ} \mathrm{C}$. After incubation, 96-well plate was washed by washing buffer once. Blocking the 96 well plate by assay buffer for $1 \mathrm{hr}$. Aspirate wells and then $100 \mu \mathrm{L}$ standards and samples were added into each well with detection antibody. The 96-well plate was incubated at $700 \mathrm{rpm}$ for 2 hours at room temperature. Aspirate wells and then wash by wash buffer for five times. The working streptavidin-HRP was added into each well and then incubated at $700 \mathrm{rpm}$ for $30 \mathrm{~min}$ at room temperature. The plate was washed for five times. TMB (3, 3', 5, 5'-tetramethylbenzidine) solution was added into each well. Stop the reaction using 2 $\mathrm{M} \mathrm{H}_{2} \mathrm{SO}_{4}$ solution. Detect the absorbance at $450 \mathrm{~nm}$ and $650 \mathrm{~nm}$ by microplate reader (BioTek, VT, USA).

\section{Statistical analysis}

All experiments were repeated at least 3 times. Results were shown as the means \pm SD. The data were analyzed by Student's unpaired T-test. The value of $p<0.05$ was indicated statistical significance.

\section{Data availability}


The datasets used and analyzed during the current study available from the corresponding author on reasonable request.

\section{Results}

EV-A71 actively replicates in PMA-primed THP-1 cells without causing significant cell death. PMA-primed THP-1 cells were seeded and infected with EV-A71 at the M.O.I. of 2 for two days. Morphological changes were observed, and no obvious cytopathic effects were observed (Figure 1A). Cell viability was examined by MTT assay, and no significant cell death appeared (Figure 1B). The expression of EV-A71 viral protein 3D was examined by Western blot analysis, and the viral protein appeared at 8 hours post-infection (p.i.) and peaked at 12 hours p.i. (Figure 1C). Total lysates of infected cells were collected, and virus titers were determined using a plaque forming assay (Figure 1D). The virus titers were increased during infection, indicating that the virus was actively replicating.

EV-A71 infection triggers the expression of proinflammatory cytokines. To determine whether EV-A71 infection can upregulate the expression of proinflammatory cytokines, total RNA extracted from EV-A71infected PMA-primed THP-1 cells was examined by RT-qPCR to detect the expression levels of TNF-a, IL-6 and IL-1 $\beta$. Our results showed that the transcripts of these three cytokines were upregulated, while no significant increase was observed in CVA16-infected cells (Figure 2A). Furthermore, nuclear parts were isolated from EV-A71-infected THP-1 macrophage-like cells. The expression of phospho-P65 in the nucleus and cytosol was examined by immunoblotting. The expression levels of phospho-P65 in the nucleus were increased at 1 hour p.i. and declined at 12 hours p.i. (Figure 2B).

EV-A71 induces the production of IL-1 $\beta$ from PMA-primed THP-1 cells in a dose-dependent manner. To determine whether IL-1 $\beta$ can be secreted, THP-1 macrophages were infected with EV-A71 or treated with LPS. Cell lysates and supernatants were collected, and pro-IL-1 $\beta$ expression was measured by Western blot analysis (Figure 3A). The amount of the secreted IL-1 $\beta$ p17 subunit was increased in a timedependent manner (Figure 3B). Supernatants were harvested at different time points and subjected to ELISA. Our results revealed that EV-A71 infection and LPS stimulation resulted in the secretion of IL-1 $\beta$ from PMA-primed THP-1 cells (Figure 3C). An increase in IL-1 $\beta$ levels was detected 12, 24, and 48 hours p.i.. To determine whether EV-A71 activates IL-1 $\beta$ in a dose-dependent manner, THP-1 macrophages were infected with EV-A71 at different M.O.I.s. The amount of secreted IL-1 $\beta$ was increased in the cells infected with EV-A71 in a dose-dependent manner (Figure 3D). Different EV-A71 strains were used to infect cells, and the expression of mature IL-1 $\beta$ was measured to determine whether the increase in secreted IL-1 $\beta$ is strain-specific. Our results revealed that all tested EV-A71 strains were able to cause the release of IL-1 $\beta$ (Figure 3E). To test whether EV-A71 infection can induce the production of IL-1 $\beta$ in human macrophages, blood samples were collected from healthy volunteers, and peripheral blood mononuclear cells (PBMCs) were isolated. Isolated PBMCs were then forced to differentiate into human macrophages. These cells were then subjected to EV-A71 infection, and our results showed that the release of mature IL-1 $\beta$ was increased at $24 \mathrm{hr}$ p.i. (Figure 3F). These findings indicated that EV-A71 can activate the production of IL$1 \beta$ in human macrocytic cells. 
Knockdown of NLRP3, RIG-I, and TLR3 decreases the expression of IL-1 $\beta$ in EV-A71-infected cells. To elucidate which types of inflammasomes are involved in EV-A71-induced IL-1 $\beta$ production, siRNAs specific for NLRP3 were transfected into THP-1 macrophages. The knockdown efficiency was confirmed by immunoblot analysis (Figure 4A). Our results also showed that the expression of NLPR3 was increased in EV-A71-infected cells. The transfected cells were subsequently infected with EV-A71 at the M.O.I. of 2, and the release of mature IL-1 $\beta$ was assessed by ELISA. The production of IL-1 $\beta$ was drastically downregulated in NLRP3 KD cells, which suggests that NLRP3 is essential for EV-A71-induced IL-1 $\beta$ production (Figure 4B). We noticed that NLRP3 KD was not able to diminish the synthesis of IL-1 $\beta$. Thus, other RNA sensors may be implicated in EV-A71-induced inflammasome formation. Previous studies suggested that RIG-I and TLR3, as well as NLRP3, are involved in inflammasome activation in influenza A virus-infected epithelial cells ${ }^{14}$. To test whether RIG-I and TLR3 were involved, siRNAs specific for these two genes were applied for cell transfection. The expression levels of RIG-I and TLR3 in scrambled siRNA- and specific siRNA-transfected THP-1 macrophages were examined by Western blot analysis (Figure $4 \mathrm{C}$ and $4 \mathrm{E}$ ). The production of IL-1 $\beta$ was determined by ELISA. Our results showed that knockdown of RIG-I and TLR3 resulted in approximately $50 \%$ downregulation of EV-A71-induced IL-1 $\beta$ release (Figure 4D and 4F).

Caspase-1 and caspase- 8 are involved in EV-A71-triggered IL-1 $\beta$ production. Caspase- 1 has been demonstrated to be involved in IL-1 $\beta$ production by promoting pro-IL-1 $\beta$ processing ${ }^{26}$. This enzyme is activated by the formation of multiple inflammasomes ${ }^{27}$. The expression of the activated caspase- 1 p20 subunit was detected in both LPS-treated and EV-A71-infected PMA-primed THP-1 cells (Figure 5A). The addition of Ac-YVAD-cmk, a caspase-1 inhibitor, suppressed EV-A71-induced IL-1 $\beta$ secretion (Figure 5B). To further characterize the role of IL-1 $\beta$ in macrophages upon EV-A71 infection, siRNA specific for caspase-1 was transfected into THP-1 macrophages that were then infected with EV-A71. The knockdown efficiency was confirmed by Western blot analysis (Figure 5C). Supernatants were harvested, and the amount of released IL- $1 \beta$ was analyzed by ELISA. The results showed that the amount of secreted IL-1 $\beta$ protein was drastically decreased in caspase-1-KD cells (Figure 5D). In addition to caspase1 , caspase-8 has also been implicated in the regulation of the NLRP3 inflammasome in murine dendritic cells ${ }^{28}$. To examine the involvement of caspase-8, PMA-primed THP-1 cells were infected with EV-A71 to induce IL-1 $\beta$ synthesis and then treated with Z-IETD-FMK. The expression of cleaved caspase- 8 was significantly decreased in infected cells treated with Z-IETD-FMK (Figure 5E). Compared to the untreated cells, only $1 / 4$ of the IL- $1 \beta$ protein was detected in the supernatant of Z-IETD-FMK-treated samples (Figure $5 F$ ). These findings suggested that both caspase- 1 and caspase- 8 are involved in IL-1 $\beta$ production in EVA71-infected THP-1 macrophages.

\section{Discussion}

Macrophages have been shown to be the target cells for many viruses, such as dengue virus and influenza virus ${ }^{29,30}$. The roles of macrophages in virus pathogenesis include altering the function of macrophages, enhancing the expression of proinflammatory cytokines, and supporting productive 
replication ${ }^{30,31}$. Prior studies showed that macrophages are productively infected by poliovirus, and these infected APCs can present the antigen ${ }^{32}$. Moreover, nonpolio enteroviruses, including CVB3 and rhinovirus, are also able to infect macrophages ${ }^{33,34}$. In this study, PMA-primed THP-1 cells, which are commonly used for studying the biological activities of macrophages, were used as an in vitro cell model. PMA primed THP-1 cells ${ }^{35}$. Our data showed that EV-A71 infection did not cause morphological changes in infected cells, and no cells were observed. This could be attributed to the enhanced expression of IFN-b in EV71-infected THP-1 macrophages (data not shown). A similar phenomenon has been demonstrated before ${ }^{35}$.

Overproduction of proinflammatory cytokines is correlated with the severity of EV-A71 infection ${ }^{2}$. Prior studies have shown that the sustained overproduction of IL-6 is correlated with disease severity in neonatal mice ${ }^{36}$. However, contrasting results have been recently published by Wang et al., who demonstrated that the use of an anti-IL-6 antibody enhances the lethality of EV-A71-infected mice ${ }^{37}$. In contrast to IL-6, several clinical reports have shown the involvement of TNF-a in the severity of illness in EV-A71 patients ${ }^{38-40}$. In accordance with previous results, our data suggest that these cells could be responsible for cytokine production ${ }^{41}$. We also showed that the expression levels of inflammatory cytokines were higher in EV-A71-infected THP-1 macrophages than in CVA16-infected cells. The differential ability of EV-A71 and CVA16 to trigger cytokine expression has been reported before by using human enteroids as a cell model ${ }^{42}$. A recent study showed that EV-A71 genotype $\mathrm{G}$, a clinical isolate from AFP patients, was able to induce higher expression of cytokine/chemokine genes than other isolates 43.

Many studies have demonstrated that EV-A71 can lead to the production of IL-1 $\beta$ via activation of the NLPR3 inflammasome ${ }^{16,44,45}$. Viral protein 2B in EMCV, PV, and EV-A71 is able to activate the NLRP3 inflammasome by inducing a flux of calcium ions ${ }^{16}$. Wang et al also demonstrated that EV-A71 induced inflammasome activation through the 3D protein ${ }^{46}$. However, the mechanism underlying inflammasome activation in EV-A71-infected THP macrophages is not clear. Our results suggest that the expression of TLR3, RIG-I, and NLRP3 is essential for EV-A71-triggered IL-1 $\beta$ release. This is the first report demonstrating that RIG-I and TLR3 are implicated in EV-A71-induced IL-1 $\beta$ release. Cytosolic RIG-I has been shown to activate inflammasomes and drive subsequent IL-1 $\beta$ production in VSV-infected human peripheral blood-derived monocytes (PBMCs) ${ }^{47}$. Furthermore, influenza A infection in human primary bronchial epithelial cells leads to IL-1 $\beta$ release through the interaction of RIG-I with ASC and caspase- $1^{14}$. It is well known that RIG-I is responsible for recognizing VSV and influenza virus, while MDA5 is related to sensing picornaviruses ${ }^{48}$. However, RIG-I is implicated in EV-A71-induced inflammasome formation but not MDA5.

Treatment with RIG-I agonists is sufficient for caspase- 1 activation and the release of mature IL-1 $\beta^{47}$. Both RIG-I and NLRP3 inflammasomes can activate caspase-1 to produce mature IL-1 $\beta$. However, AcYVAD-cmk, a caspase 1 inhibitor, was not able to totally abolish the production of IL-1 $\beta$ in EV-A71- 
infected THP-1 macrophages. Moreover, treatment with a caspase-8 inhibitor drastically downregulated the release of IL-1 $\beta$. Therefore, both caspase- 1 and caspase- 8 are involved in virus-triggered inflammasome activation. Caspase-8 can serve as a positive regulator of the NLRP3 inflammasome to improve IL-1 $\beta$ maturation in mouse dendritic cells ${ }^{28}$. Furthermore, activated caspase 8 can act on pro-IL$1 \beta$ directly to form mature IL-1 ${ }^{49}$. Many studies have demonstrated that caspase 8 is involved in the production of IL-1 $\beta$ in innate immune cells ${ }^{50,51}$. Our results suggest that more than one caspase is involved in EV-A71-induced IL-1 $\beta$ production in THP-1 macrophages. However, the underlying mechanisms are not clear. In summary, this study shows that EV-A71 can induce the upregulation of IL$1 \beta$ with the involvement of TLR3, NLRP3, and RIG-I.

\section{Declarations}

\section{Acknowledgements}

This work was financially supported by the Research Center for Emerging Viral Infections from The Featured Areas Research Center Program within the framework of the Higher Education Sprout Project by the Ministry of Education (MOE) in Taiwan and the Ministry of Science and Technology (MOST), Taiwan (MOST 110-2634-F-182-001). This work was also supported by Ministry of Science and Technology (MOST), Taiwan (MOST 110-2320-B-182-020) and Chang Gung Memorial Hospital, Taiwan (CMRPD1K0701, BMRP-B33).

\section{Author contributions}

H.I.H. conceived and supervised the experiments, funding acquisition. C.C.C., J.Y.L., C.J.C., C.J.L. and L.S.Y. performed the experiments and data analysis. $\mathrm{HIH}, \mathrm{CCC}$ : wrote the manuscript draft. All authors reviewed the manuscript.

\section{Competing interests}

The authors declare that they have no conflict of interests.

\section{References}

1. Solomon, T. et al. Virology, epidemiology, pathogenesis, and control of enterovirus 71. Lancet Infect Dis 10, 778-790, doi:10.1016/s1473-3099(10)70194-8 (2010).

2. Ye, N. et al. Cytokine responses and correlations thereof with clinical profiles in children with enterovirus 71 infections. BMC Infect Dis 15, 225, doi:10.1186/s12879-015-0965-1 (2015).

3. Zhu, X. et al. Pyroptosis induced by enterovirus A71 infection in cultured human neuroblastoma cells. Virology 521, 69-76, doi:10.1016/j.virol.2018.05.025 (2018).

4. Feng, M. et al. Mechanism for the lethal effect of enterovirus A71 intracerebral injection in neonatal mice. Lab Invest 100, 596-605, doi:10.1038/s41374-019-0351-5 (2020). 
5. Arango Duque, G. \& Descoteaux, A. Macrophage cytokines: involvement in immunity and infectious diseases. Front Immunol 5, 491, doi:10.3389/fimmu.2014.00491 (2014).

6. Klepper, A. \& Branch, A. D. Macrophages and the Viral Dissemination Super Highway. EC Microbiol 2, 328-336 (2015).

7. Cheung, C. Y. et al. Induction of proinflammatory cytokines in human macrophages by influenza A (H5N1) viruses: a mechanism for the unusual severity of human disease? Lancet 360, 1831-1837, doi:10.1016/s0140-6736(02)11772-7 (2002).

8. Negash, A. A. et al. IL-1 $\beta$ production through the NLRP3 inflammasome by hepatic macrophages links hepatitis C virus infection with liver inflammation and disease. PLoS Pathog 9, e1003330, doi:10.1371/journal.ppat.1003330 (2013).

9. Hernandez, J. C., Latz, E. \& Urcuqui-Inchima, S. HIV-1 induces the first signal to activate the NLRP3 inflammasome in monocyte-derived macrophages. Intervirology 57, 36-42, doi:10.1159/000353902 (2014).

10. Kim, B. S. et al. IL-1 signal affects both protection and pathogenesis of virus-induced chronic CNS demyelinating disease. J Neuroinflammation 9, 217, doi:10.1186/1742-2094-9-217 (2012).

11. Ribeiro, D. E. et al. Hyperactivation of P2X7 receptors as a culprit of COVID-19 neuropathology. Mol Psychiatry 26, 1044-1059, doi:10.1038/s41380-020-00965-3 (2021).

12. Shrivastava, G., León-Juárez, M., García-Cordero, J., Meza-Sánchez, D. E. \& Cedillo-Barrón, L. Inflammasomes and its importance in viral infections. Immunol Res 64, 1101-1117, doi:10.1007/s12026-016-8873-z (2016).

13. Kanneganti, T. D. Central roles of NLRs and inflammasomes in viral infection. Nat Rev Immunol 10, 688-698, doi:10.1038/nri2851 (2010).

14. Pothlichet, J. et al. Type I IFN triggers RIG-I/TLR3/NLRP3-dependent inflammasome activation in influenza A virus infected cells. PLoS Pathog 9, e1003256, doi:10.1371/journal.ppat.1003256 (2013).

15. Chivero, E. T. et al. HIV-1 Tat Primes and Activates Microglial NLRP3 Inflammasome-Mediated Neuroinflammation. J Neurosci 37, 3599-3609, doi:10.1523/jneurosci.3045-16.2017 (2017).

16. Ito, M., Yanagi, Y. \& Ichinohe, T. Encephalomyocarditis virus viroporin 2B activates NLRP3 inflammasome. PLoS Pathog 8, e1002857, doi:10.1371/journal.ppat.1002857 (2012).

17. He, X. et al. NLRP3-dependent pyroptosis is required for HIV-1 gp120-induced neuropathology. Cell Mol Immunol 17, 283-299, doi:10.1038/s41423-019-0260-y (2020).

18. Chang, Y. S. et al. SARS Unique Domain (SUD) of Severe Acute Respiratory Syndrome Coronavirus Induces NLRP3 Inflammasome-Dependent CXCL10-Mediated Pulmonary Inflammation. Int J Mol Sci 21, doi:10.3390/ijms21093179 (2020).

19. Ribeiro, D. E. et al. Hyperactivation of P2X7 receptors as a culprit of COVID-19 neuropathology. Mol Psychiatry 26, 1044-1059, doi:10.1038/s41380-020-00965-3 (2021). 
20. Kanneganti, T. D. et al. Critical role for Cryopyrin/Nalp3 in activation of caspase-1 in response to viral infection and double-stranded RNA. J Biol Chem 281, 36560-36568, doi:10.1074/jbc.M607594200 (2006).

21. Bao, J., Sun, T., Yue, Y. \& Xiong, S. Macrophage NLRP3 inflammasome activated by CVB3 capsid proteins contributes to the development of viral myocarditis. Mol Immunol 114, 41-48, doi:10.1016/j.molimm.2019.07.012 (2019).

22. Robinson, K. S. et al. Enteroviral $3 \mathrm{C}$ protease activates the human NLRP1 inflammasome in airway epithelia. Science 370, doi:10.1126/science.aay2002 (2020).

23. Wang, C. et al. NLRP3 deficiency exacerbates enterovirus infection in mice. Faseb j 33, 942-952, doi:10.1096/fj.201800301RRR (2019).

24. Wang, H. et al. Reciprocal Regulation between Enterovirus 71 and the NLRP3 Inflammasome. Cell Rep 12, 42-48, doi:10.1016/j.celrep.2015.05.047 (2015).

25. Wang, Y. et al. Pyroptosis induced by enterovirus 71 and coxsackievirus B3 infection affects viral replication and host response. Sci Rep 8, 2887, doi:10.1038/s41598-018-20958-1 (2018).

26. Schroder, K. \& Tschopp, J. The inflammasomes. Cell 140, 821-832, doi:10.1016/j.cell.2010.01.040 (2010).

27. Wu, J., Fernandes-Alnemri, T. \& Alnemri, E. S. Involvement of the AIM2, NLRC4, and NLRP3 inflammasomes in caspase-1 activation by Listeria monocytogenes. J Clin Immunol 30, 693-702, doi:10.1007/s10875-010-9425-2 (2010).

28. Antonopoulos, C. et al. Caspase-8 as an Effector and Regulator of NLRP3 Inflammasome Signaling. J Biol Chem 290, 20167-20184, doi:10.1074/jbc.M115.652321 (2015).

29. Kyle, J. L., Beatty, P. R. \& Harris, E. Dengue virus infects macrophages and dendritic cells in a mouse model of infection. J Infect Dis 195, 1808-1817, doi:10.1086/518007 (2007).

30. Cline, T. D., Beck, D. \& Bianchini, E. Influenza virus replication in macrophages: balancing protection and pathogenesis. J Gen Virol 98, 2401-2412, doi:10.1099/jgv.0.000922 (2017).

31. Yu, W. C. et al. Viral replication and innate host responses in primary human alveolar epithelial cells and alveolar macrophages infected with influenza H5N1 and H1N1 viruses. Journal of virology 85, 6844-6855, doi:10.1128/jvi.02200-10 (2011).

32. Wahid, R., Cannon, M. J. \& Chow, M. Dendritic cells and macrophages are productively infected by poliovirus. Journal of virology 79, 401-409, doi:10.1128/jvi.79.1.401-409.2005 (2005).

33. Benkahla, M. A. et al. Coxsackievirus-B4E2 can infect monocytes and macrophages in vitro and in vivo. Virology 522, 271-280, doi:10.1016/j.virol.2018.06.010 (2018).

34. Laza-Stanca, V. et al. Rhinovirus replication in human macrophages induces NF-kappaB-dependent tumor necrosis factor alpha production. Journal of virology 80, 8248-8258, doi:10.1128/jvi.0016206 (2006).

35. Chanput, W., Mes, J. J. \& Wichers, H. J. THP-1 cell line: an in vitro cell model for immune modulation approach. Int Immunopharmacol 23, 37-45, doi:10.1016/j.intimp.2014.08.002 (2014). 
36. Khong, W. X., Foo, D. G., Trasti, S. L., Tan, E. L. \& Alonso, S. Sustained high levels of interleukin-6 contribute to the pathogenesis of enterovirus 71 in a neonate mouse model. Journal of virology 85 , 3067-3076, doi:10.1128/jvi.01779-10 (2011).

37. Wang, L. C. et al. Suppression of interleukin-6 increases enterovirus A71 lethality in mice. J Biomed Sci 24, 94, doi:10.1186/s12929-017-0401-5 (2017).

38. Duan, G. et al. Serum inflammatory cytokine levels correlate with hand-foot-mouth disease severity: a nested serial case-control study. PloS one 9, e112676, doi:10.1371/journal.pone.0112676 (2014).

39. Zheng, W. et al. Alteration of serum high-mobility group protein 1 (HMGB1) levels in children with enterovirus 71-induced hand, foot, and mouth disease. Medicine (Baltimore) 96, e6764, doi:10.1097/md.0000000000006764 (2017).

40. Sun, J. F., Li, H. L. \& Sun, B. X. Correlation analysis on serum inflammatory cytokine level and neurogenic pulmonary edema for children with severe hand-foot-mouth disease. Eur J Med Res 23, 21, doi:10.1186/s40001-018-0313-1 (2018).

41. Gong, X. et al. Excessive proinflammatory cytokine and chemokine responses of human monocytederived macrophages to enterovirus 71 infection. BMC Infect Dis 12, 224, doi:10.1186/1471-2334-12224 (2012).

42. Zhao, X. et al. Human Intestinal Organoids Recapitulate Enteric Infections of Enterovirus and Coronavirus. Stem Cell Reports 16, 493-504, doi:10.1016/j.stemcr.2021.02.009 (2021).

43. Mohanty, M. C., Varose, S. Y. \& Saxena, V. K. Susceptibility and cytokine responses of human neuronal cells to multiple circulating EV-A71 genotypes in India. Sci Rep 11, 17751, doi:10.1038/s41598-021-97166-x (2021).

44. Triantafilou, K., Kar, S., van Kuppeveld, F. J. \& Triantafilou, M. Rhinovirus-induced calcium flux triggers NLRP3 and NLRC5 activation in bronchial cells. Am J Respir Cell Mol Biol 49, 923-934, doi:10.1165/rcmb.2013-00320C (2013).

45. Wang, Y., Gao, B. \& Xiong, S. Involvement of NLRP3 inflammasome in CVB3-induced viral myocarditis. Am J Physiol Heart Circ Physiol 307, H1438-1447, doi:10.1152/ajpheart.00441.2014 (2014).

46. Wang, W. et al. EV71 3D Protein Binds with NLRP3 and Enhances the Assembly of Inflammasome Complex. PLoS Pathog 13, e1006123, doi:10.1371/journal.ppat.1006123 (2017).

47. Poeck, H. et al. Recognition of RNA virus by RIG-I results in activation of CARD 9 and inflammasome signaling for interleukin 1 beta production. Nat Immunol 11, 63-69, doi:10.1038/ni.1824 (2010).

48. Pichlmair, A. \& Reis e Sousa, C. Innate recognition of viruses. Immunity $27,370-383$, doi:10.1016/j.immuni.2007.08.012 (2007).

49. Bossaller, L. et al. Cutting edge: FAS (CD95) mediates noncanonical IL-1 $\beta$ and IL-18 maturation via caspase-8 in an RIP3-independent manner. J Immunol 189, 5508-5512, doi:10.4049/jimmunol.1202121 (2012).

50. Gaidt, M. M. et al. Human Monocytes Engage an Alternative Inflammasome Pathway. Immunity 44, 833-846, doi:10.1016/j.immuni.2016.01.012 (2016). 
51. Maelfait, J. et al. Stimulation of Toll-like receptor 3 and 4 induces interleukin- 1 beta maturation by caspase-8. J Exp Med 205, 1967-1973, doi:10.1084/jem.20071632 (2008).

\section{Figures}

\section{Figure 1}

THP-1 macrophages are permissive to EV-A71 infection. THP-1 macrophages were infected with EV-A71 at the M.O.I. of 2. (A)The morphologies of infected cells were observed using inverted microscopy and images were taken at 12, 24, and 48 hours post-infection. (magnification $=200 \mathrm{X}$ ). (B) Cell viability of EVA71-infected THP-1 macrophages were examined by MTT assay. (C) Total protein was extracted from Mock- and EV-A71-infected cells and then subjected to Western blot to examine the expression of viral protein 3D. The expression of b-actin was used as an internal control. (D) Total cellular lysates were prepared from EV-A71-infected THP-1 macrophages and the amounts of viral particles were determined by plaque assay.

\section{Figure 2}

EV-A71 infection induces pro-inflammatory cytokine production in THP-1 macrophages. (A) PMA-primed THP-1 cells were infected with EV-A71 or CVA16 at an M.O.I. of 2. The total RNA was isolated, then the genes and viral RNA 5'UTR were analyzed by RT-qPCR. (B) THP-1 macrophages were infected with EV$A 71$ at the M.O.I. of 2. Nuclear and cytosol proteins were harvested and the expression levels of p-P65 were detected by immunoblot. The expression of lamin and b-actin was used internal controls, respectively. 
Figure 3

A

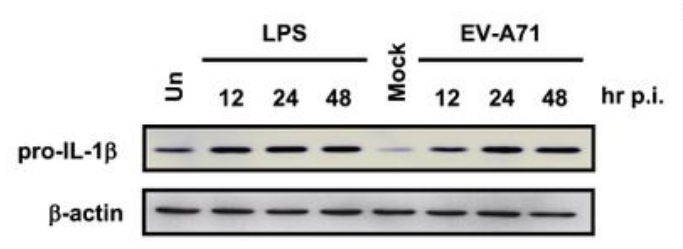

B

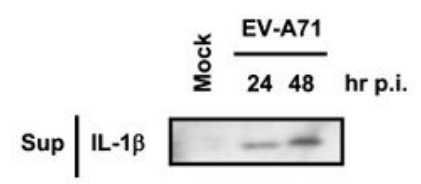

C

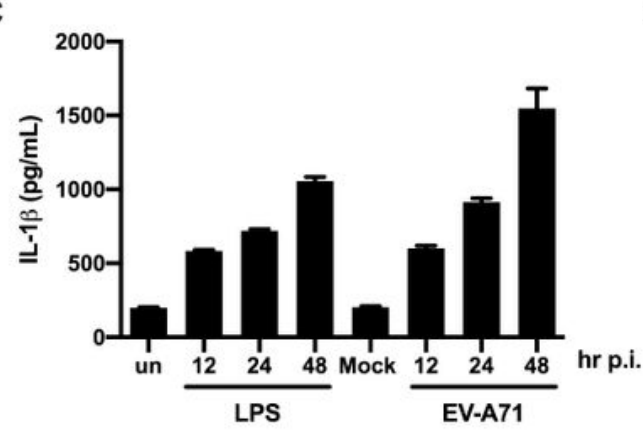

D

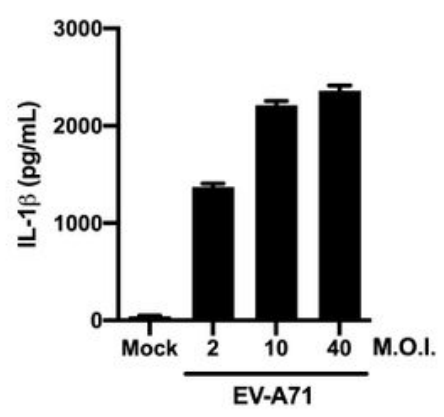

$\mathbf{E}$

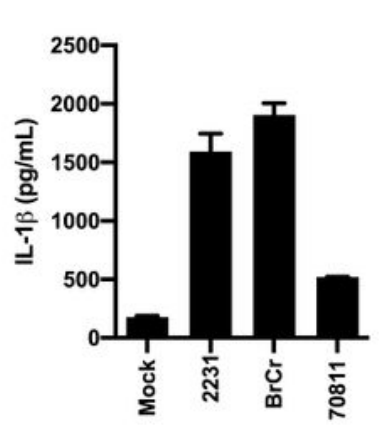

$\mathbf{F}$

PBMC derived Macrophage

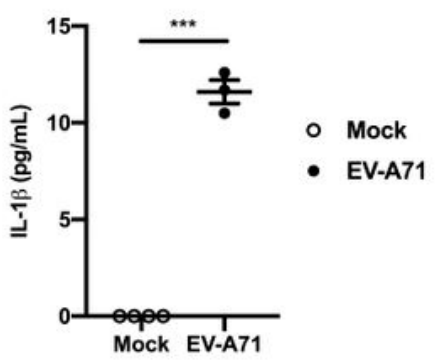

\section{Figure 3}

EV-A71 infection induces IL-1b production in human macrophages. (A) THP-1 cells were differentiated with PMA for 48 hours and then infected with EV-A71 at the M.O.I. of 2. Cell lysates and supernatants were collected at 12, 24, and $48 \mathrm{hr}$ p.i. and subjected to immunoblot analysis to detect the expression of pro-IL-1b. The expression of b-actin was used as an internal control. (B) PMA primed THP-1 cells were infected with EV-A71 (2 M.O.I.). The supernatants were collected to detect the expression levels of IL-1b 
by immunoblot. (C) Supernatants were then harvested to examine the secreted IL-1b levels by ELISA. (D) PMA differentiated-THP-1 macrophages were infected with EV-A71 at M.O.I. of 2, 10, and 40.

Supernatants were collected and expressed IL-1b amounts were examined by ELISA. (E) PMA-primed THP-1 cells were infected with EV-A71 (2 M.O.I., strain of 2231, BrCr and 70811), and the expression of IL$1 \beta$ in supernatant was assayed by ELISA. (F) Human PBMCs were differentiated into macrophages $(n=3)$ and infected with EV-A71 (10 M.O.I.), the expression levels of secreted IL-1b protein were analyzed. ( $\star \star \star p<~$ 0.001 Student's unpaired T-test)

\section{Figure 4}

NLRP3, RIG-I, and TLR3 regulates the EV-A71-triggerd IL-1b production. (A) NLRP3 siRNA (siNLRP3) (100 $\mathrm{nM}$ ) or scrambled siRNA (Sc) was transfected into PMA-primed THP-1 cells, then infected with EV-A71 at the M.O.I. of 2. The expression levels of NLRP3 were detected by Western blot. (B) The secreted IL-1b was measured by ELISA. (C) 100 nM siRNA target RIG-I (siRIG-I) was transfected into PMA-primed THP-1 cells for 48 hours, then infected with 2 M.O.I. EV-A71 for 12, 24 hours, the expression levels of RIG-I and viral protein VPO were examined by Western blot. (D) Supernatants were harvested and the secreted IL-1b protein was assayed by ELISA. (E) Specific TLR3 siRNA (siTLR3) and scramble siRNA was transfected into PMA-primed THP-1 cells for 48 hours. Cells were then infected with 2 M.O.I. EV-A71 for 12 hours, and the knockdown efficiency was confirmed by Western blot analysis. $(F)$ The supernatants were collected to

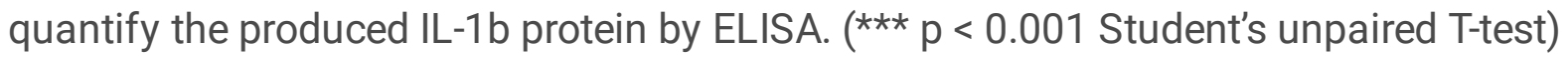

\section{Figure 5}

EV-A71 induced IL-1b production is dependent on caspase-1 and caspase-8 activation. (A) PMA-primed THP-1 cells were treated with LPS $(1 \mu \mathrm{g} / \mathrm{mL})$ or infected with EV-A71 (2 M.O.I.), and the expression levels of cleaved caspase-1 p20 were analyzed by Western blot. (B) PMA-primed THP-1 cells were pre-treated with $1 \mathrm{mM}$ Ac-YVAD-cmk for 1 hour, then infected with 2 M.O.I. EV-A71 in the presence or absence of AcYVAD-cmk, and assayed the expression levels of secreted IL-1b by ELISA. (C) THP-1 macrophages were transfected with scrambled siRNA or caspase-1 siRNA (siCASP1) for 48 hours and then infected with EVA71 at the M.O.I. of 2. The expression levels of caspase-1 and EV-A71 VP0 protein were assessed by immunoblot. The expression of b-actin was used as an internal control. (D) The supernatants were harvested at 12 and $24 \mathrm{hr}$ p.i. and the secreted IL-1b levels were examined by ELISA. (E) THP-1 macrophages were infected with EV-A71 at an M.O.I. of 2 and then treated with Z-IETD-FMK. The protein 
expression of caspase-8 was confirmed by Immunoblot. (F) the amount of IL-1b in supernatant was measured by ELISA. (***, $p<0.001$ Student's unpaired T-test)

\section{Supplementary Files}

This is a list of supplementary files associated with this preprint. Click to download.

- rawdataofimmunoblot.docx 